\title{
Miranda
}

Revue pluridisciplinaire du monde anglophone /

Multidisciplinary peer-reviewed journal on the English-

speaking world

19 | 2019

Rethinking Laughter in Contemporary Anglophone Theatre

\section{Jean-Pierre Richard, Shakespeare Pornographe. Un théâtre à double fond}

\section{Armelle Sabatier}

\section{OpenEdition}

\section{Journals}

Édition électronique

URL : http://journals.openedition.org/miranda/21548

DOI : $10.4000 /$ miranda. 21548

ISSN : 2108-6559

\section{Éditeur}

Université Toulouse - Jean Jaurès

Édition imprimée

Date de publication : 7 octobre 2019

\section{Référence électronique}

Armelle Sabatier, « Jean-Pierre Richard, Shakespeare Pornographe. Un théâtre à double fond ». Miranda

[En ligne], 19 | 2019, mis en ligne le 14 octobre 2019, consulté le 16 février 2021. URL : http:// journals.openedition.org/miranda/21548; DOI : https://doi.org/10.4000/miranda.21548

Ce document a été généré automatiquement le 16 février 2021.

\section{(c) (i) () $\Theta$}

Miranda is licensed under a Creative Commons Attribution-NonCommercial-NoDerivatives 4.0 International License. 


\title{
Jean-Pierre Richard, Shakespeare Pornographe. Un théâtre à double fond
}

\author{
Armelle Sabatier
}

\section{RÉFÉRENCE}

Jean-Pierre Richard, Shakespeare Pornographe. Un théâtre à double fond (Paris, Éditions Rue d'Ulm, 2019), 244 p., ISBN : 978-2-7288-0622-5

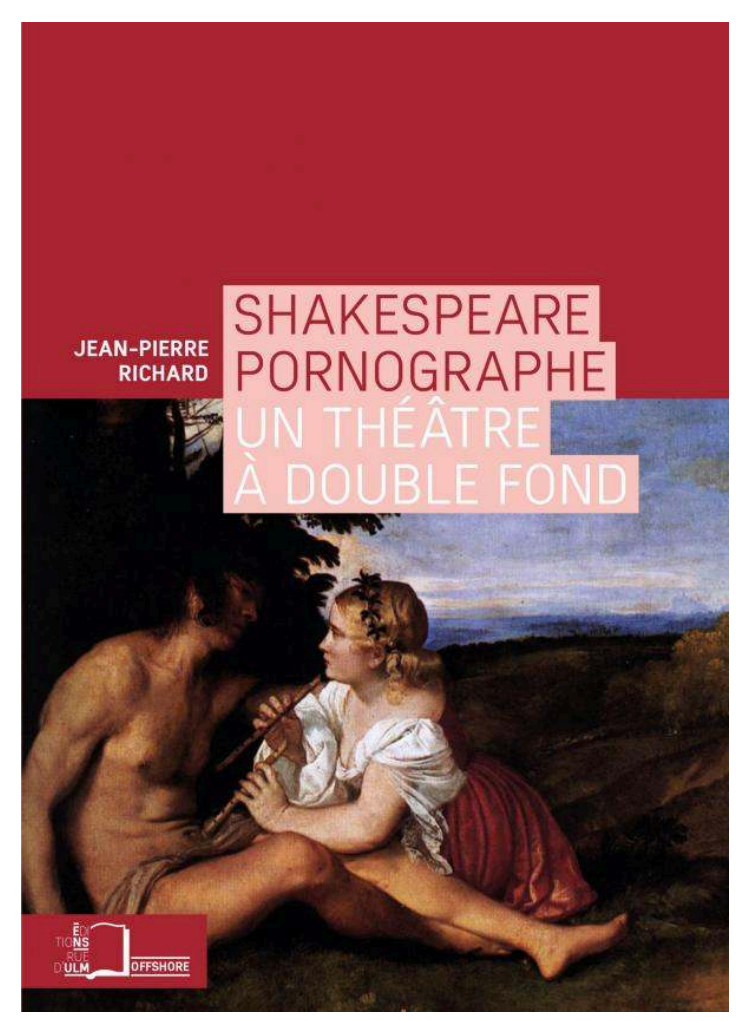


1 Érotique ou pornographique? Telle est la question abordée, sans détour, par JeanPierre Richard, dans son dernier ouvrage consacré à l'un des plus grands monuments de la littérature anglaise: William Shakespeare. Éminent traducteur, lauréat de plusieurs prix de traduction (prix Maurice-Edgar Coindreau en 1983 et Grand Prix Halpérine-Kaminsky en 1992), cet universitaire s'est confronté à des auteurs aussi divers que Peter Gill, Robert Holman, Bill Morrison ou encore le poète Samuel Taylor Coleridge. Il participe, depuis plusieurs années, à la traduction des œuvres théâtrales de Shakespeare pour la prestigieuse édition de la Pléiade. Dès les premières lignes de son livre, Jean-Pierre Richard avoue que les pièces de Shakespeare s'avèrent tout simplement «intraduisibles». Et pour cause, les textes de Shakespeare, selon lui, reposent sur un double discours, présentent deux théâtres où foisonnent en permanence des sous-entendus salaces et grivois. L'écriture "pornographique» de Shakespeare, que Jean-Pierre Richard étudie au sens étymologique du terme ( $«$ du grec gaphein ("écrire») et pornè ("courtisane ») : il écrit sur la prostituée » p.11), est présente dans tous les genres dramatiques abordés par ce dramaturge et poète dans sa longue carrière. L'auteur de ce volume invite, ainsi, ses lecteurs à « un voyage au pays de l'obscène », et leur propose une « odyssée de la grivoiserie » (p.12).

2 Cet ouvrage tente de faire revivre ce théatre perdu que nos contemporains, d'après l'auteur, n'entendent pas ou plus. Jean-Pierre Richard signale que les éditeurs ne veulent pas voir ou laisser paraître "ce » Shakespeare, comme si certains voulaient préserver l'image d'un Shakespeare poète de l'«amour romantique ", du moins en France. Cette réticence vis-à-vis du double mouvement du texte trouve son origine au $\mathrm{XIX}^{\text {ème }}$ siècle, une période où les textes de Shakespeare ont subi une véritable censure, autant dans l'Angleterre victorienne que dans la France de Victor Hugo. Cependant, parler de l'obscène chez Shakespeare en 2019 n'est pas vraiment un sujet nouveau, comme le rappelle l'auteur lui-même. La critique anglo-saxonne a, depuis longtemps, transgressé cette forme de tabou universitaire et littéraire autour du Shakespeare pornographe, et ce, dès 1945 avec l'ouvrage d'Eric Partridge, Shakespeare's Bawdy. Pour autant, dans sa thèse consacrée au désir dans la poésie élisabéthaine, en particulier le premier poème narratif de Shakespeare, Venus and Adonis (1593), Laetitia Sansonetti signale que la publication universitaire de Partridge « avait été assimilée [à l'époque] à de la littérature érotique ${ }^{1}$ ». L'adjectif "bawdy» en anglais n'est pas non plus l'équivalent de pornographique ${ }^{2}$. Un nombre important de publications sur ce sujet met en avant, encore aujourd'hui, le caractère érotique et non pornographique des textes de Shakespeare. En outre, cette forme de pruderie a longtemps prévalu pour les Sonnets de Shakespeare, comme le rappelle brillamment Katherine Duncan-Jones dans l'introduction de son édition des sonnets ${ }^{3}$. Le dictionnaire de Frankie Rubinstein, Dictionary of Shakespeare's Sexual Puns and their Significance, publié en 1984, a battu en brèche ces résistances, en proposant une étude très détaillée et minutieuse des innombrables jeux de mots salaces, des double (voir triple) entendre qui émaillent les textes de Shakespeare. D'ailleurs, les éditions anglo-saxonnes contemporaines des textes de Shakespeare signalent toujours les jeux de mots grivois, et ce grâce, entre autres, au travail colossal de Rubinstein. Néanmoins, Jean-Pierre Richard rappelle qu'aucune étude de cette ampleur consacrée à la nature pornographe du théâtre de Shakespeare n'existe en France. Ainsi, cet ouvrage cherche à remettre en question l'image encore vivace, en France, d'un Shakespeare «fantasmé », d'un Shakespeare qui serait, selon l'expression consacrée, "politiquement et moralement correct ». 
3 Les cinq chapitres qui structurent le livre dévoilent les multiples facettes de cette écriture et de cette esthétique du pornographique, toujours sous une plume brillante. Pour illustrer ses propos, l'auteur s'appuie, à chaque fois, sur des passages précis, présentés d'abord dans le texte original et suivis de la, ou plutôt des traductions. D'ailleurs, l'auteur prévient ses lecteurs, dès l'introduction, du double mouvement de son propre texte entre la langue anglaise et le français, un bilinguisme qui pourrait "gêner la lecture" d'un lectorat peu familier de la langue de Shakespeare. Or, ce passage incessant d'une langue à une autre constitue une des intérêts majeurs de cette étude. La «double» traduction donnée pour un seul passage dans une pièce met en avant les différentes strates de sens sur lesquelles Shakespeare joue en permanence. La polysémie, l'homophonie ainsi que d'autres figures de rhétorique et de style permettent de créer un texte réversible, à la fois sérieux et jovial, grivois et tragique. Cette pornographie sous-jacente, qui reste « de papier », pour reprendre les termes de l'auteur, dévoile l'hypocrisie ou la fausse innocence de certains personnages shakespeariens: par exemple, après le chapitre un, le lecteur ne percevra plus Desdémone comme une jeune femme sage et pieuse. L'auteur poursuit sa réflexion « en joignant le geste à la parole » dans le chapitre deux, consacré à la mise en scène et au jeu des acteurs dans l'espace scénique: la fameuse scène de la fente dans le mur, un élément du décor que l'acteur doit représenter sur scène dans Le Songe d'une nuit d'été, donne lieu, bien entendu, à une analyse des plus savoureuses. Les chapitres suivants abordent des questions liées à la réception, à la parodie et aux débats littéraires de l'époque. Le dernier chapitre propose un portrait des mœurs et de la société élisabéthaines et du monde du théâtre, fort utile pour les non spécialistes de la Renaissance anglaise.

4 Ainsi cet ouvrage ne manquera pas de captiver les lecteurs par son style brillant, sa grande érudition et ses analyses très fines du texte et de la représentation théâtrale. Les lecteurs français découvriront assurément un "autre Shakespeare", comme le qualifie l'auteur; les lecteurs anglicistes ou anglicisants ne bouderont pas leur plaisir face à la virtuosité du traducteur qu'est Jean-Pierre Richard. La jovialité et la grivoiserie qui se dégagent de ce texte tendent, cependant, à faire oublier les aspects plus sombres de la sexualité que Shakespeare explore dans ses œuvres poétiques, un « autre Shakespeare » qui pourrait faire l'objet d'un prochain livre....

\section{NOTES}

1. Je tiens à remercier Laetitia Sansonetti de m'avoir permis de lire sa thèse : «Représentations $\mathrm{du}$ désir dans la poésie élisabéthaine (Venus and Adonis, Hero and Leander, The Faerie Queene II et III », Thèse soutenue le 8 novembre 2011, Université Sorbonne Nouvelle - Paris III, p.211 n.29.

2. Laetitia Sansonetti aborde les différences entre l'érotisme et le pornographique, en particulier dans son analyse pp.210-211.

3. The Arden Shakespeare, Londres, Bloomsbury, 2013. 
INDEX

Mots-clés : William Shakespare, théâtre, traduction, pornographie, censure

Keywords : censorship, drama, pornography, William Shakespeare, translation

\section{AUTEURS}

ARMELLE SABATIER

Maître de Conférences

Université Paris II Panthéon - Assas

arm.saba@free.fr 